\title{
Benign cystic tumor of the mandible. A case report and literature review
}

\author{
Tahura Khwaja*,1, Kaveri Hallikeri ${ }^{1}$, Sahana Adirajaiah², Venkatesh Anehosur ${ }^{2}$, \\ Pragati Rai ${ }^{1}$
}

${ }^{1}$ Department of Oral Pathology, SDMCDSH, Dharwad, Karnataka, India, ${ }^{2}$ Department of Oral and Maxillofacial Surgery, SDMCDSH, Dharwad, Karnataka, India

\begin{abstract}
The keratocystic odontogenic tumor (KCOT), earlier considered as the odontogenic keratocyst (OKC), is now classified as a benign neoplasm owing to its aggressive nature which includes a high recurrence rate. This case report describes a symptomatic large intraosseous odontogenic cystic neoplasm in the left mandible presenting radiographically as a large multilocular radiolucency and associated with an impacted tooth. The typical clinical and distinguishing histological features of KCOT morphed by the presence of inflammation are discussed here together with an intriguing clear cell component and epithelial theques and their clinical relevance. The newly acknowledged neoplastic potential serving significant role in treatment planning has also been addressed. Various lesions can present intraosseously as large multilocular radiolucencies in the jaws. Histopathological examination remains the mainstay for the diagnosis of such lesions.
\end{abstract}

Keywords: keratocystic odontogenic tumor; odontogenic keratocyst; benign cystic tumor, multilocular radiolucency

\section{Introduction}

The keratocystic odontogenic tumor (KCOT) was formerly known as the 'odontogenic keratocyst' (OKC) as coined by Philipsen in 1956 [1]. The new designation is a clearer implication to its neoplastic nature. In its classification of head and neck tumors in 2005, the World Health Organization (WHO) included this lesion in the category of benign tumors that show odontogenic epithelium and mature, fibrous stoma without odontogenic ectomesenchyme [2]. The change in terminology was centered on the behavior of OKC as a neoplasm and not a benign

Received: November 2016; Accepted after review: February 2017; Published: March 2017.

*Corresponding author: Dr. Tahura Khwaja,

MDS, Oral and Maxillofacial Pathology \&

Microbiology, HHF-46, HIG Building, Pundalik Nagar,

Porvorim, Goa 403521, India.

Phone: +917722091706

E-mail: tahura.khwaja@gmail.com cystic lesion, given its local destructive nature and a high recurrence rate.

The present case report highlights a large benign odontogenic tumor of the mandible associated with an impacted tooth with evidence of epithelial theques and focal areas of clear cells, while presenting its characteristic cystic nature superimposed by inflammation. A note on its differential diagnoses has also been made.

\section{Case report}

A 27 year-old female presented in our hospital with pain and swelling in the lower left back tooth region of jaw which was present for a duration of two months. The swelling was initially small and gradually increased in size with history of pus discharge from the same region. She gave no history of habits, and her medical and familial histories were noncontributory. 
On examination, a diffuse, firm swelling was noted extraorally in the left lower half of the face with an intact overlying skin. A solitary left submandibular lymph node was palpable, firm and tender. Intraoral examination revealed a diffuse swelling measuring $25 \times 15 \mathrm{~mm}$ in the buccal aspect of the mandible on the left side, extending from the region of the second premolar till the missing third molar region, and causing buccal vestibular obliteration. The first molar was grossly carious (Figure 1). The mucosa overlying the swelling was intact and normal colored. Pus discharge was however, noted in the third molar region. On palpation, the swelling was tender, soft in consistency, with an egg-shell crackling of left buccal cortical plate. Routine blood investigation values were within normal limits.

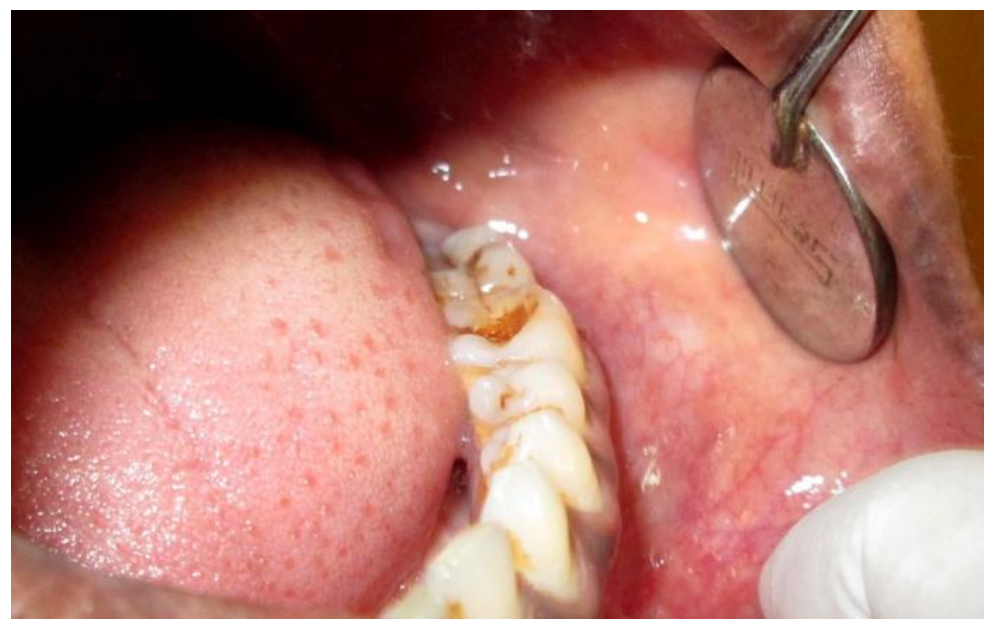

Fig. 1. Clinical presentation: Diffuse intra-oral swelling in the posterior mandible on the left side causing buccal vestibular obliteration. A grossly carious of the first molar is also noted.

Panoramic radiography revealed a welldefined multilocular radiolucency with a radiopaque corticated margin in the left mandible extending from the mesial aspect of 36 till the ramus posteriorly. A horizontally impacted 38 within this radiolucency as well as apical resorption of both the roots of 36 and 37 , and thinning of the inferior border of the mandible (Figure 2) were evident. The lesion was presumed to be a unicystic ameloblastoma and differential diagnoses of dentigerous cyst and KCOT were given.

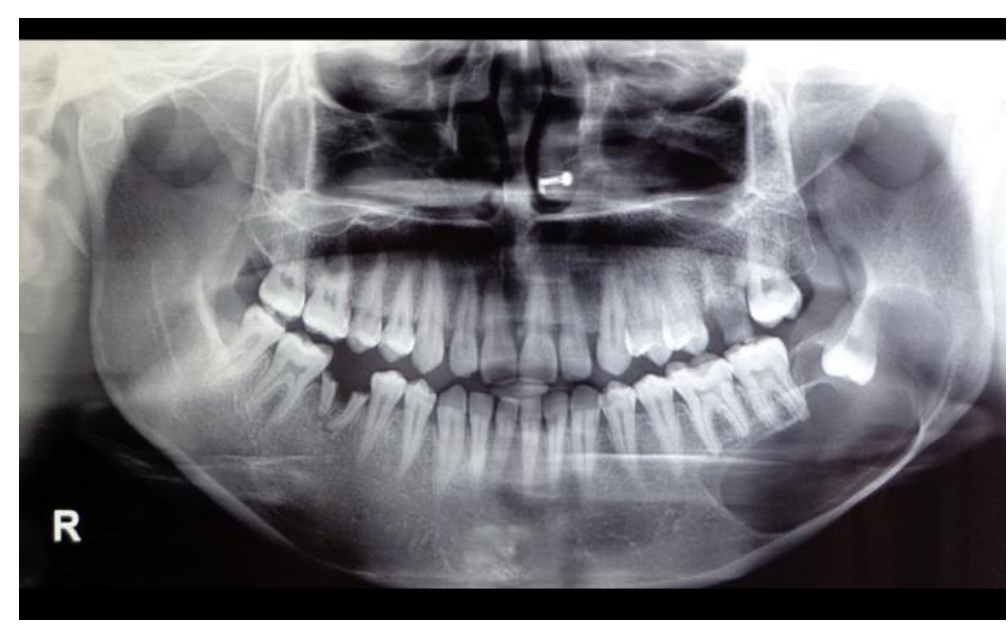

Fig. 2. Panoramic radiography: A well-defined multilocular radiolucency is noted in the left mandible with an impacted third molar within. 
Aspiration of the lesional contents revealed a greenish odorless fluid. The cytosmear however, was non-contributory as it revealed only few squamous cells and occasional foamy histiocytes. An incisional biopsy procedure was carried out. Microscopy of the hematoxylin and eosin (HE) stained sections revealed a cystic lumen lined by parakeratinized stratified squamous epithelium and bound by a condensed hyalinized to thickly fibrous capsular stroma. Stromal areas closer to the luminal surface showed few dilated and engorged capillaries with few inflammatory cells. A focal area with cholesterol clefts was also evident. A final diagnosis of an infected KCOT was made and a month later the lesion was enucleated in toto under general anesthesia followed by chemical cauterization by of the cystic cavity with Carnoy's solution. All the three molars in the left mandible were extracted during the procedure. Intra-operatively, bony perforation of the buccal cortical plate by the cyst was noted (Figure 3 ) and the cystic cavity showed presence of pus. The lesion was submitted for microscopic evaluation.

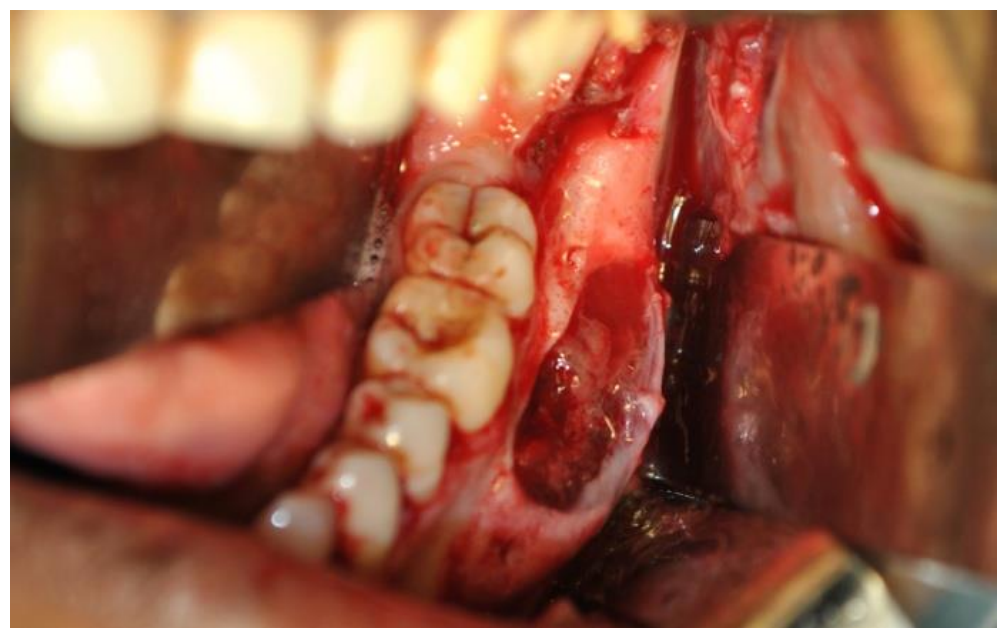

Fig. 3. Intra-operative picture: Evidence of expansion and perforation of the buccal cortical plate by the cyst.

The gross specimen was a soft grayishbrown mass with a smooth well-defined capsule and a cystic lumen and measured 22 x $20 \mathrm{~mm}$. Histological examination of the HE stained sections demonstrated a cystic lumen lined by a corrugated parakeratinized epithelial lining of varying thickness and bound by a fibrous to fibro-cellular connective tissue capsule. Distinctive focal areas of palisaded hyperchromatic basal cells as well as areas of basal cell hyperplasia were noted. The epithelio-mesenchymal interface was flat in most of the areas, showing tendency for detachment from the capsule, but focal areas of epithelial theques and proliferation of the epithelium by the formation of rete ridges were evident. This proliferation was thought to be secondary to the chronic inflammation in the underlying connective tissue stroma (Figure 4). A mass of cholesterol clefts, multinucleated giant cells, and mixed inflammatory cell infiltrate were noted with few cholesterol clefts within the thickened epithelium (Figure 5). An interesting feature was the evidence of a focal area with clear cells in the thickened epithelium which was negative for Periodic acid Schiff (PAS) stain. The connective tissue capsule subjacent to these clear cells was devoid of inflammation (Figure 6). The cystic capsule also showed capillaries and hemorrhage.

The wound healed by secondary intention. The patient was followed up for four months and showed no signs of recurrence. 

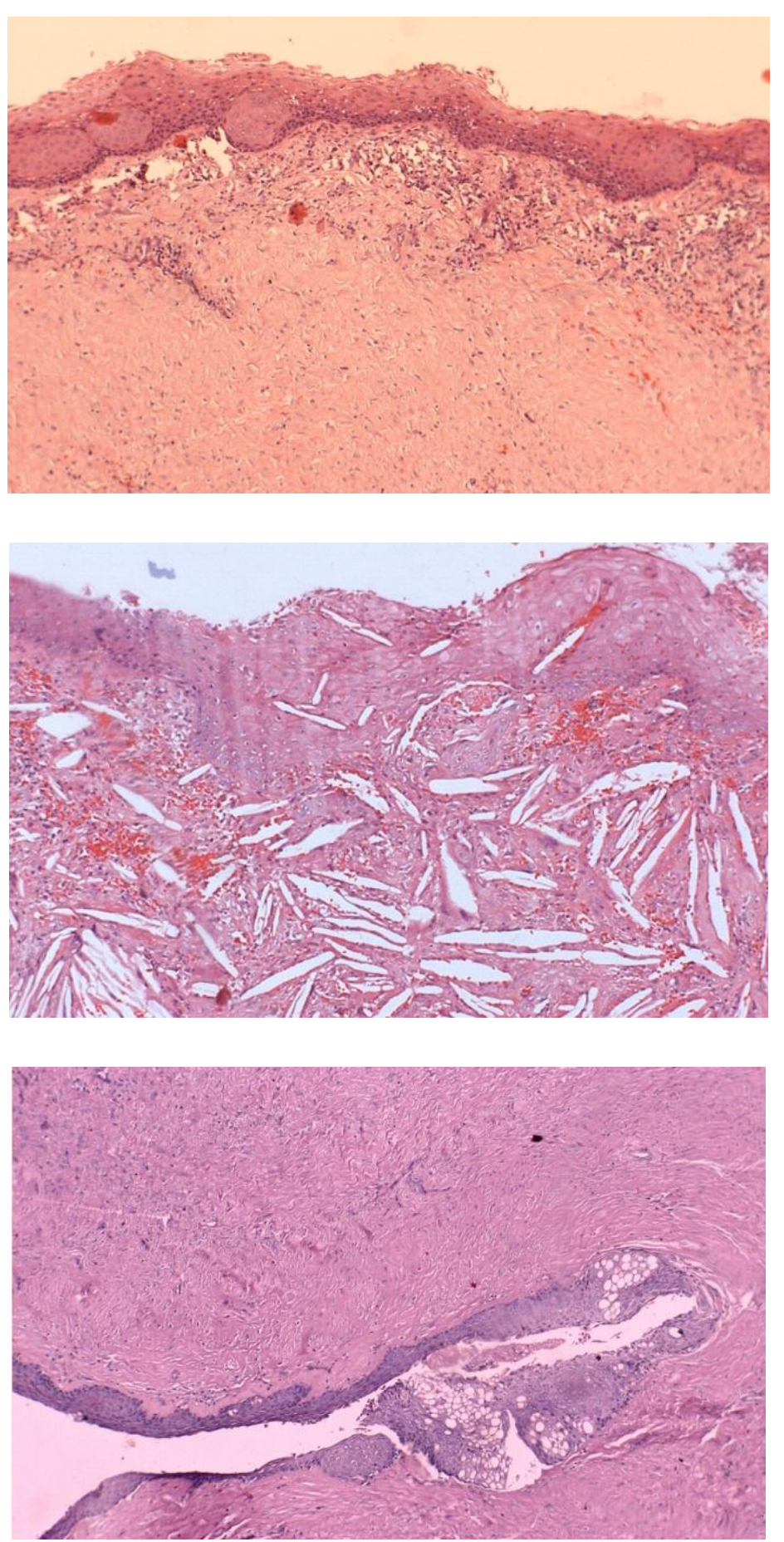

Fig. 4. Microscopic view of excisional specimen: cystic lumen lined by parakeratinized epithelium showing numerous theques and bound by a fibrous to fibro-cellular connective tissue capsule with subepithelial chronic inflammation (HE, $\mathrm{x} 100)$.

Fig. 5. Microscopic view of excisional specimen: connective tissue capsule showing cholesterol clefts, multinucleated giant cells, and mixed inflammatory cell infiltrate. Few cholesterol clefts are noted within the thickened parakeratinized epithelium (HE, x100).

Fig. 6. Microscopic view of excisional specimen: focal area of thickened epithelium with clear cells (PAS, x100).

\section{Discussions}

KCOT accounts for $10 \%$ of all jaw cysts [3]. This entity presents with many distinctive clinical and histological features. KCOT encompasses a wide age range, the peak being second and third decades, with a predilection for white males. The mandible shows a higher rate of involvement (65-83\% of cases) than the maxilla, with a predilection for the third molar-ramus-angle region [2]. In
$25 \%$ to $40 \%$ of the cases there is association of an unerupted tooth in the lesion [4]. KCOT is generally regarded as an intraosseous lesion; however, rare peripheral cases have been reported. Majority of the cases comprise the gingival or alveolar mucosa in the caninepremolar region. Multiple lesions are commonly associated with the Nevoid basal cell carcinoma syndrome (NBCCS) or the Gorlin syndrome [4]. 
Most of the KCOTs are asymptomatic and are mostly detected in the late stage when they have reached a large size $[5,6]$. Signs and symptoms of pain, swelling, expansion, drainage and bony perforation, may lead to its diagnosis or it may be found incidentally during routine radiographic examination. On occasion, pain, swelling, and drainage enunciates a secondary infection of the cyst as was noted in the present case wherein the infectious etiology was attributed to the grossly carious first molar.

KCOTs present radiographically as either unilocular $(83.5 \%$ cases) or multilocular $(16.5 \%$ cases $)$ radiolucencies with welldemarcated sclerotic margins [4]. The lesion's proximity to the tooth may cause displacement and/or root resorption, although displacement $(28.3 \%)$ is more commonly seen than resorption (5\%) [7]. Unilocular lesions can be located surrounding the crown of unerupted or impacted tooth, simulating dentigerous cysts. Larger lesions, like in the current case, usually appear multilocular. Multilocular or large unilocular KCOTs can be indistinguishable from ameloblastoma or its unicystic variant respectively. KCOT may show similarity to an odontogenic myxoma because of the mild expansion and multilocular appearance [5].

The KCOT characteristically grows in an antero-posterior direction within medullary bony cavity without causing much expansion $[4,5]$, a feature that helps differential clinical and radiographic diagnosis of dentigerous cyst or ameloblastoma of comparable size which are usually associated with bony expansion. Complete radiographic examination is essential in suspicion of KCOT because of its aggressive behavior and high recurrence rate. Advanced imaging techniques like computerized tomography, cone-beam computerized tomography, and digitalized scans are useful in assessment of cortical perforation and involvement of the soft tissue. The definitive diagnosis, however, relies on histological examination.

The KCOT comprises a spectrum of histopathological variants; single typical cystic lesion, single cystic lesion having multiple satellite microcysts, and solid or multicystic variant. The classic histopathologic features of the KCOT are distinctive, but may be altered by inflammation. It is characterized by a uniformly thin, six to eight celled epithelial lining, which does not demonstrate rete ridges. This may result in the epithelium separating from the underlying fibrous connective tissue forming a cleft. The epithelium also shows a well-defined, palisaded basal layer made of hyperchromatic columnar to cuboidal cells. The luminal surface displays a usually corrugated parakeratotic epithelial layer with frequent presence of a keratinous material within the lumen. The connective tissue capsule surrounding the epithelial lining is usually thin with relatively few cells widely separated by the mucopolysaccharide-rich stroma [1-4].

In addition to the typical features, the present case also revealed theques of epithelium as well as a focal area with clear cells in the thickened epithelium. Clear cells are characteristic cellular components of odontogenic cysts like lateral periodontal cyst and gingival cyst of the adult, or may occur in odontogenic tumors such as ameloblastoma, calcifying odontogenic cyst, and calcifying epithelial odontogenic tumor. Their presence in odontogenic cysts and tumors has been related to their derivation from the remnants of the dental lamina and rest of Malassez which are known to have glycogen-rich clear cells as a typical feature $[3,8]$. Some authors, however, report that the presence of clear cells may indicate increased tumor aggressiveness $[9,10]$ and thus their presence should underscore the tumor conversion to malignancy. Only one case of solid KCOT with clear cells has been reported wherein the clear cells were arranged in cords in a small focal area. Existence of diastase-labile PAS-positive material, indicative of glycogen, supported that clear cells were of odontogenic origin with of no significance to tumor aggressiveness in the stated case [11]. In our case the clear cells showed negative staining reaction with PAS stain.

Epithelial thickenings or theques formation may represent the recapitulation of odontogenic epithelium to ontogeny under pathological condition. The possible mode of formation of epithelial plaques is by the localized proliferation of the basal cells. Cysts most commonly showing such epithelial 
theques are the gingival cyst of the adult, lateral periodontal cyst, botryoid odontogenic cyst, and sialodontogenic cyst [3].

Several theories of the development of the KCOT have been presented. They are presumed to arise from the remnants of the dental lamina which persist in the oral tissues after the completion of odontogenesis, or from the basal cell layer of the oral mucosal epithelium. The etiology of KCOTs is strongly linked to mutation of tumor-suppressor PTCH gene mapped to the chromosome 9q22.3-q31 [3].

The treatment of the KCOT stands controversial as a middle ground is to be achieved between minimizing the possibility of recurrence and detriment of an extensive resection. Multiple treatment modalities have been proposed and employed. These include conservative approach like decompression alone, simple enucleation with or without curettage, or marsupialization as well as aggressive treatment like peripheral ostectomy, chemical curettage with Carnoy's solution, and resection. There is however, no consensus on adequate or appropriate treatment of this lesion; a case-wise management of KCOT is therefore essential $[3,5]$.

\section{Conclusions}

KCOT is often preliminarily diagnosed as less aggressive cyst, which could erroneously receive more conservative surgical treatment, paving a path for an increased tendency to recurrence. Careful analysis of each case,

\section{References}

1. Rajendran R, Sivapathasundharam B. Shafer's textbook of Oral Pathology. New Delhi: Elsevier, 2012.

2. Philipsen HP. Keratocystic odontogenic tumor. In: Barnes L, Evenson JW, Reichart P, Sidransky D, editors. World Health Organization classification of tumors. Pathology and genetics of head and neck tumors. Lyon: IARC Press; 2005:306-307.

3. Shear M, Speight PM. Odontogenic keratocyst. In: Shear M, Speight PM, editors. Cysts of the taking into account the clinical behavior, radiographic features, and incisional biopsy for histopathological evaluation can help select more suitable treatment modalities. The need for a thorough follow-up cannot be underestimated and must range for 5 to 10 years.

The evaluation of CD44v6 immunoexpression in cervical carcinomas is useful for the assessment of tumor response to neoadjuvant therapy and of tumor aggressive behavior. The high level of $\mathrm{E}$ cadherin immunoexpression in tumors associated with neoadjuvant therapy reflects its involvement in the prevention of HPV oncoproteins action, with benefits on the outcome and NAT efficiency. The increase of E-cadherin cytoplasmic immunoexpression is correlated with tumor aggressiveness and with a favorable response to neoadjuvant therapy. The increase of the batch of patients and the use of a panel of antibodies may provide an accurate characterization of the tumor response to neoadjuvant therapy and validate its prognosis value.

\section{Acknowledgement}

We would like to thank Dr. Srinath Thakur, Principal, Sri Dharmasthala Manjunatheshwara College of Dental Sciences, Karnataka, for his constant support.

\section{Competing interests}

The authors declare that they have no competing interest.
Oral and Maxillofacial Regions, Oxford: Blackwell Munksgaard; 2007:6-58.

4. Neville BW, Damm DD, Allen CM, Bouquot JE. Oral \& Maxillofacial Pathology. $3^{\text {rd }}$ ed. Noida: W B Saunders; 2002.

5. White SC, Pharoah MJ. Oral radiology: principles and interpretation. $6^{\text {th }}$ ed. St. Louis: Mosby; 2009.

6. Cawson RA, Odell EW. Cawson's essentials of oral pathology and oral medicine. $7^{\text {th }}$ ed. London: Churchill Livingstone; 2002. 
7. Haring JI, Van Dis ML. Odontogenic keratocysts: a clinical, radiographic, and histopathologic study. Oral Surg Oral Med Oral Pathol 1988; 66:145-153.

8. Reichart PA, Philipsen HP. Odontogenic Tumors and Allied Lesions. $1^{\text {st }}$ ed. London: Quintessence Publishing Co. Ltd.; 2004.

9. Waldron CA, Small IA, Silverman H. Clear cell ameloblastoma-an odontogenic carcinoma. J Oral Maxillofac Surg 1985; 43(9):707-717.
10. Rangel AL, da Silva AA, Ito FA, Lopes MA, de Almeida OP, Vargas PA. Clear cell variant of calcifying epithelial odontogenic tumor: is it locally aggressive? J Oral Maxillofac Surg 2009; 67(1):207-211.

11. Kawano K, Okamura K, Kashima K, et al. Solid variant of keratocystic odontogenic tumor of the mandible: report of a case with a clear cell component and review of the literature. Oral Surg Oral Med Oral Pathol Oral Radiol 2013; 116:393-398. 